\title{
Histopathology of salivary glands
}

\author{
F. Carubbi ${ }^{1,2}$, A. Alunno ${ }^{3}$, R. Gerli ${ }^{3}$, R. Giacomelli ${ }^{2}$ \\ ${ }^{1}$ Department of Medicine, ASL1 Avezzano Sulmona L'Aquila; \\ ${ }^{2}$ Rheumatology Unit, Department of Biotechnological and Applied Clinical Science, University of L'Aquila, Italy; \\ ${ }^{3}$ Rheumatology Unit, Department of Medicine, University of Perugia, Italy
}

\section{SUMMARY}

Salivary gland (SG) biopsy is a technique broadly applied for the diagnosis of primary Sjögren's syndrome (pSS), lymphoma accompanying SS, sarcoidosis, amyloidosis, and IgG4-related disease The most peculiar feature of pSS on biopsy is focal lymphocytic sialadenitis. In the past, several histological scores have been reported in the literature to describe glandular involvement during pSS. However, the variability among centres in reporting glandular scores is one of the rationales behind the development of standardised consensus guidance. SGs as well as lacrimal glands are involved in up to $50 \%$ of patients with IgG4-related disease with 3 histopathological hallmarks such as dense lymphoplasmacytic infiltration, storiform fibrosis and obliterative phlebitis. SGs can be also affected by amyloidosis with MSG biopsy being more sensitive than that of rectal mucosa or subcutaneous fat. SG involvement is a rare manifestation during sarcoidosis, and the presence of non-caseating granulomas needs to be differentiated from granulomas of other etiology. This review article provides an overview of normal and pathological SGs in the context of rheumatic diseases, identifying key elements in the tissue as diagnostic and prognostic biomarkers, useful in the current clinical practice.

Key words: Primary Sjögren's syndrome; IgG4; Amyloidosis, Sarcoidosis.

Reumatismo, 2018; 70 (3): 146-154

\section{INTRODUCTION}

alivary glands (SGs) are organs able to secrete a watery substance which is important for several physiological functions, including the protection of teeth and surrounding soft tissues and the lubrication of the oral cavity, the latter being crucial for speech and perception of food taste. Major SGs include the left and right parotid, submandibular and sublingual glands, while about 600 to 1000 minor SGs (labial, buccal, lingual, palatal, retromolar) are widely distributed in the oral cavity and oropharynx, lying above the muscle layer and separated from the oral mucous membrane by a thin layer of fibrous connective tissue (1). These exocrine glands produce saliva from a secretory structure, called salivary acinus, through a duct system. Based on the type of secretion, there are three main type of acini: serous, mucous and mixed. SG histopathology is considered the gold standard for the diagnosis of primary Sjögren's syndrome (pSS); moreover, SG biopsy can be used to stage the disease, provide prognostic data, and monitor disease change upon therapeutic intervention. Besides pSS, SG biopsy has been employed for the diagnosis of other systemic disorders, such as amyloidosis, sarcoidosis, IgG4-related disease, and neonatal hemochromatosis. The purpose of this review article is to provide an overview on current knowledge about the diagnostic and prognostic role of SG histopathology in rheumatology practice.

\section{SJÖGREN'S SYNDROME}

\section{Diagnostic role and classification}

pSS is a chronic inflammatory disease which mainly affects exocrine glands (2). SG biopsy is widely used in the classification of pSS according to the AmericanEuropean Consensus Group classification criteria (3) and the most recent American College of Rheumatology/European League against Rheumatism (ACR/EULAR) criteria (4). Although integrated as classification criteria, SG biopsy is widely 
used to support a diagnosis of pSS, and in centres where biopsy is frequently undertaken, as many as $40 \%$ of cases are autoantibody negative (5). In order to obtain MSG, a lip biopsy is usually performed although parotid gland biopsy may be also performed. In particular, Pijpe et al. analyzing concurrent parotid and MSG biopsies in 30 patients investigated for pSS, concluded that the diagnostic sensitivity and specificity were identical and the presence of inflammatory infiltrate and germinal centre-like structures were similar (6). The histopathological hallmark of pSS MSG is the focal lymphocytic sialadenitis (FLS) $(7,8)$, characterized by the presence of one or more dense aggregates with 50 or more lymphocytes (focus), usually located in the perivascular or periductal areas, with sensitivity and specificity $>80 \%$ and high positive predictive value (9). These peculiar features make MSG biopsy particularly helpful to evaluate patients with non-specific clinical manifestations, extra-glandular involvement, very early disease, and/ or autoantibody negativity. However, FLS may occur in conjunction with other autoimmune diseases, infectious diseases (e.g. hepatitis $\mathrm{C}$ virus, human immunodeficiency virus) and in healthy individuals, so it is not by itself diagnostic for pSS (10-12). The spectrum of MSG histopathological damage ranges from mild to diffuse infiltrates with progressive loss of normal glandular tissue. T-cells predominate in mild lesions, whereas B-cells are the most represented cell subset in the advanced lesions (13-15). Other cell types such as macrophages, dendritic cells (DC), plasmacytoid dendritic cells (pDC) and natural-killer (NK) cells account for less than $10 \%$ of the total cell population (16). Infiltrating lymphocytes are often organized into tertiary ectopic lymphoid structures, showing a network including specific segregated T- and B-cell zones associated with follicular dendritic cells (fDCs). Some of these ectopic lymphoid structures mirror classic germinal centres (17). In the past, several histological scores have been reported in the literature to describe glandular involvement during pSS (Table I). The Chisholm and
Table I - Histological patterns and scoring systems in minor salivary gland biopsy. For detailed information concerning each scoring system, please refer to the manuscript and corresponding references.

\begin{tabular}{|l|l|l|l|}
\hline $\begin{array}{l}\text { Chisholm \& Mason } \\
\text { grading system }\end{array}$ & $\begin{array}{l}\text { Tarpley } \\
\text { score }\end{array}$ & $\begin{array}{l}\text { Severity of } \\
\text { inflammatory infiltrate }\end{array}$ & $\begin{array}{l}\text { Histological } \\
\text { pattern }\end{array}$ \\
\hline 0 & & 1 & Normal \\
\cline { 1 - 3 } 1 & 0 & 2 & NSCS \\
2 & & 3 & SCS \\
\hline & & 4 & \\
3 & 1 & & FLS \\
4 & 2 & 5 & \\
\hline
\end{tabular}

NSCS, non-specific chronic sialadenitis; SCS, sclerosing chronic sialadenitis; FLS, focal lymphocytic sialadenitis. For detailed information concerning each scoring system, please refer to the manuscript and corresponding references.

Mason grading system considers MSGs with normal architecture (grade 0), slight and moderate infiltrate (grades 1 and 2, respectively), presence of 1 focus (grade 3), and more than 1 focus in $4 \mathrm{~mm}^{2}$ (18). The Tarpley score highlights only the absence (score 0 ) or the presence (scores 1-4) of foci in $4 \mathrm{~mm}^{2}$ of glandular tissue, with the higher scores identifying glands with many foci and consequent progressive derangement of glandular tissue (19). Finally, in order to assess and quantify the presence of inflammatory cells, some authors proposed an inflammatory severity score including:

1) normal MSGs (score 1);

2) MSGs with different amounts of inflammatory cells (scores 2-4);

3 ) the presence of 1 or more foci in $4 \mathrm{~mm}^{2}$ (score 5) $(20,21)$.

In addition to FLS, in accordance with the Sjögren's International Clinical Collaborative Alliance (SICCA) protocol, different morphologic patterns of chronic inflammation commonly occur in MSG biopsy specimens: non-specific chronic sialadenitis (NSCS) and chronic sclerosing sialadenitis (SCS) (22) (Figure 1). Features of NSCS and SCS such as atrophy and duct dilation or parenchymal fibrosis are common in the population and so may coexist with pSS. Unfortunately, the SICCA protocol does not include additional information concerning size and degree of organisation of foci, presence of germinal centres and lymphoepithelial lesions, the latter being 
characterised by lymphocytic infiltration of ducts and basal cell hyperplasia resulting in a multilayered epithelium, and which may also have prognostic significance. However, the lymphoepithelial lesions are more commonly observed in parotid tissue than in MSGs (6), and lymphoma occurs more often in parotid tissue than in MSGs. Recently, the Sjögren's histopathology workshop group from the EULAR Sjögren's syndrome study group (eSSential) provided standardized consensus guidance for the use of labial SG histopathology in the classification of pSS and in clinical trials (23). The variability among centres in reporting glandular scores is one of the rationales behind the development of this consensus (24). Authors have focused this work on labial SG tissue, as this remains the most commonly employed in clinical practice (23). Briefly, given the scattered nature of foci, good quality glandular tis- sue of a minimum of 4 labial SGs with a total glandular surface area of at least 8 $\mathrm{mm}^{2}$ were proposed to allow a robust and reliable analysis. This is due to the stochastic nature of foci in FLS, since the examination of an insufficient glandular area may lead to an underestimation or overestimation of the focus score (FS). The FS is defined as the total number of foci in the specimen divided by the glandular surface area and multiplied by 4 . Therefore, FS is the number of foci per $4 \mathrm{~mm}^{2}$ of glandular tissue. To avoid bias, FS calculation should include the whole of the glandular surface area in the denominator, including abnormal areas. Above an FS of 10 , foci are typically confluent, giving an arbitrary score of 12 . FLS cannot be attributed when the histological appearance of the glands is dominated by features associated with NSCS or SCS, with no evidence of any foci adjacent to normal parenchyma;

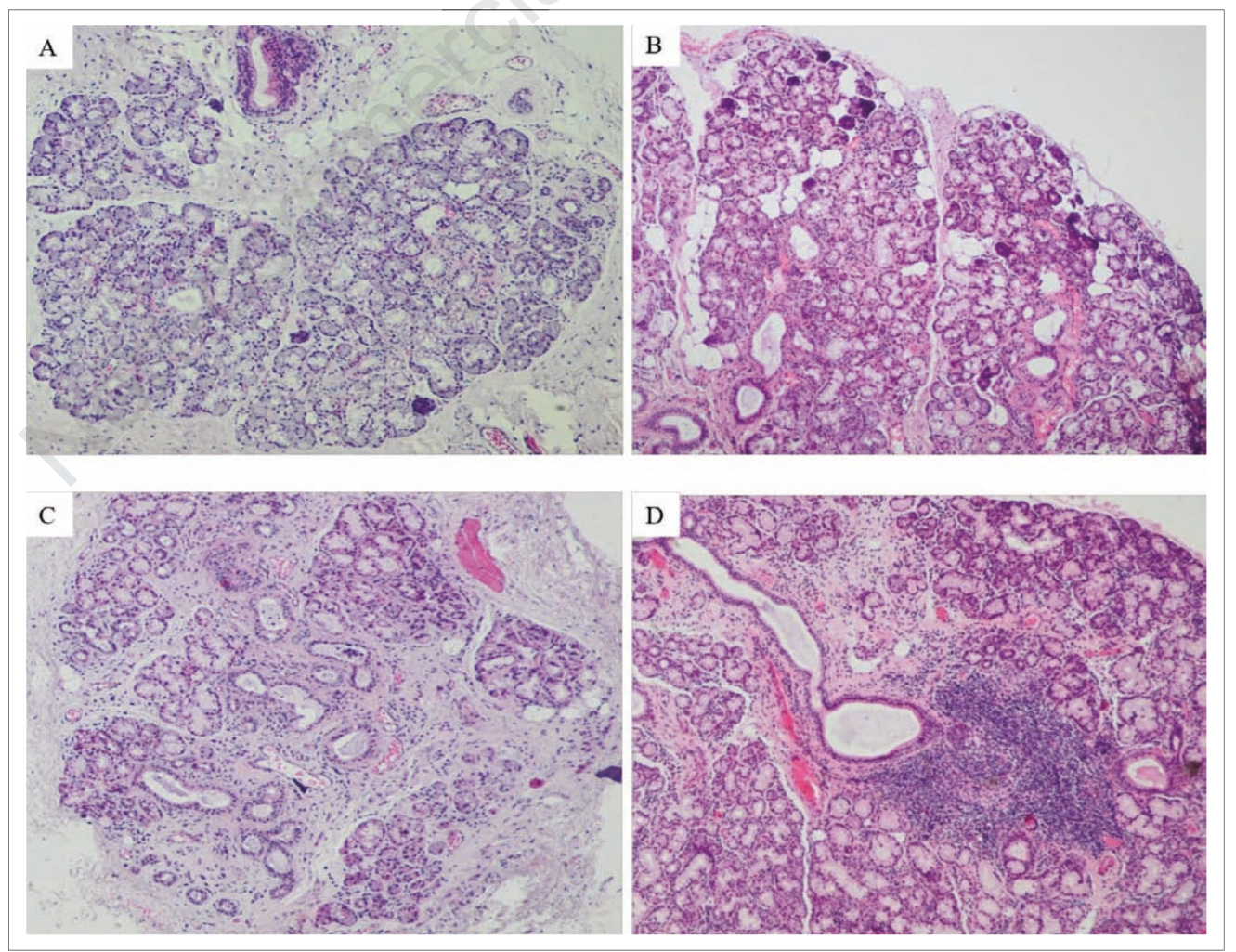

Figure 1 - Hematoxylin-eosin staining of minor salivary glands biopsies at $4 \mathrm{x}$ magnification: A) normal; B) non-specific chronic sialadenitis; C) sclerosing chronic sialadenitis; D) focal lymphocytic sialadenitis. For detailed information concerning each histological pattern, please refer to the manuscript. 
therefore, extent (absent, mild, moderate, severe) of atrophy, fibrosis and duct dilatation, should be reported. There is strong agreement that the presence of germinal centre-like structures should be reported in routine practice (23). Previous studies reported prevalence of germinal centres in pSS patients from $18 \%$ to $67 \%(25-27)$ and germinal centre formation is associated with higher FS (28). Haematoxylin and eosin (H\&E) staining is considered sufficient to allow accurate detection of a fully formed germinal centre by experienced pathologists, in particular in secondary lymphoid organs with well-distinguished light and dark zone segregation. The dark zone is the area of centroblast proliferation, while centrocytes, $\mathrm{T}$ follicular helper cells and fDCs with their large dendriticlike cytoplasm constitute the light zone. However, in MSGs, this detection is more challenging and germinal centre-like structures are often only appreciable as a lighter area within the follicular structure without the classical dark/light zone segregation. On this basis, additional immunostaining to assess ectopic lymphoid structures has been suggested, including B-cell lymphoma (Bcl)-6 (29), CD21 and activationinduced cytidine deaminase (AID) (30), without agreement on staining protocols to identify ectopic lymphoid structures. Bcl6 is a transcription factor highly expressed by all germinal centre B-cells; CD21 is a marker of fDCs and, although CD21+ fDC networks are a prerequisite for germinal centre development, their presence does not imply that germinal centres are present; AID is expressed if the germinal centres are functional but accurate staining for this is technically challenging. Moreover, the combination of a double staining for CD3 and CD20 to identify B/T cell segregation would reduce the risk of germinal centre-like structure overestimation if relying on $\mathrm{CD} 21$ alone $(26,27,31)$. Given the invasive nature of MSG biopsy, alternative methodologies in order to study salivary gland pathology are evaluated. Ultrasound has been the most studied imaging modality, but diagnosis agreement with biopsy seems only modest with a lower sensitivity
(32). Serum or glandular cytokine profiles have been reported to distinguish between patients with and without germinal centrelike structures on biopsy, but is not applicable in clinical practice $(26,33)$. Although salivary biomarkers show promise, the relationship between saliva proteomics and histopathological features requires further evaluation (34).

\section{Prognostic value}

A correlation between infiltrate severity and different lymphocytic populations has been described in the literature, emphasizing the fact that distinct immune responses most likely mediate the clinical diversity of pSS phenotype (13). In mild infiltrates, the presence of T cells is evident, while more advanced infiltrates are dominated by $\mathrm{B}$ cells. However, the cellular composition of the MSG inflammatory infiltrates of pSS patients seems to remain unchanged from the time of diagnosis (14) and characterizes certain patient subgroups, implying that different pathogenic mechanisms underlie in distinct clinical subgroups. Among infiltrate features, the number and/or proportion of B cells may be of particular interest, for at least two reasons:

1) the proportion of B cells is highest in advanced lesions, increases with histopathological scores, and severe inflammatory lesions characterized by B cell predominance have been associated with systemic and extra-glandular manifestations, including lymphoma (35);

2) the B-cell burden within the glands might influence the efficacy of anti-Bcell drugs such as rituximab (RTX).

In particular, the baseline number of $\mathrm{CD} 20^{+} \mathrm{B}$ cells $/ \mathrm{mm}^{2}$ of parotid parenchyma may serve as a prognostic biomarker to predict response to RTX treatment (27), although another study concluded that a high proportion of MSG B cells predicted an absence of a clinical response to RTX (36). It is highly likely that differences in assessment of patients' responsiveness to RTX treatment by using different methods and techniques lead to different results. Several studies investigated the use of FS not only for diagnosis but also for progno- 
sis in pSS patients. A higher FS predicts a greater decline in unstimulated salivary flow over time (37), and FS has also been reported to correlate moderately with stimulated salivary flow (38). Risselada and colleagues showed that a lymphocytic FS $\geq 3$ was associated with an increased risk for lymphoma, highlighting the prognostic value of this histological feature to predict lymphoma and other adverse outcomes at the time of diagnosis in a cohort of 174 patients (28). Recently, in a large cohort of $383 \mathrm{pSS}$ patients, we demonstrated that patients with higher FS were more likely to present with salivary gland swelling, lymphoma and, from a serological point of view, hypocomplementemia, particularly a reduction of $\mathrm{C} 4$, hypergammaglobulinemia, circulating monoclonal component and double association anti-Ro/SSA plus anti-La/SSB (39). This observation may confirm the link between B-cell hyperactivation, immune complexes production and consequent serum complement consumption, with a higher level of lymphocytic organization at the target tissue of pSS (40). Detection of germinal centres in the lymphoid infiltrates of the salivary glands is clinically relevant, because patients with germinal centres are diagnosed with $\mathrm{pSS}$ at a younger age (26), the presence of these structures is associated with more severe disease, including B-cell hyperactivation features $(25,26)$, and may be considered a predictor of lymphoma development (30, 35). However, recently, Haacke and Colleagues reported that in their cohort of pSS patients, germinal centre presence in labial gland biopsies is not a predictive factor for pSS-associated parotid mucosa-associate lymphoid tissue (MALT) lymphomas (41). Such discrepancies may be due to the different histological approach in the detection of the germinal centre (42) or might be related to histopathological differences between minor and parotid salivary gland biopsies, although a previous study in a small cohort of patients did not report a difference in numbers of germinal centres (6). However, it should be noted that most saliva is produced by the major salivary glands and only $5-10 \%$ is produced by the $600-1000$
MSGs (1), and that the predominant parenchymal cells in parotid are serous acinar cells, while those in MSGs are mucous acinar cells. Moreover, Pijpe et al. reported a lower incidence of lymphoepithelial lesions in MSGs compared with major glands (6). As mentioned above, it has been suggested that lymphoepithelial lesions may also be associated with lymphoma development (11). Taking into account data concerning the prognostic role of SG biopsy, an objective biomarker of glandular inflammation would be desirable as a validated outcome measurement. However, in the absence of therapeutic strategies definitively proven to alter disease activity in pSS, the ability of MSG biopsy to be an effective biomarker remains unclear. We reported a reduction in FS following RTX (43). RTX treatment in $\mathrm{pSS}$ leads to a major reduction of lymphocytic infiltration and to fewer B cells, germinal centres and lymphoepithelial lesions also in parotid gland parenchyma (27). In a pilot study, abatacept seems to be able to reduce the absolute number of foci but not foci per $\mathrm{mm}^{2}$ (44). Despite so many years of use and the amount of studies in literature, is hard to determine the precise value of SG biopsy in pSS, due to the lack of data regarding the natural history of the histopathological changes associated with pSS, the test reliability or repeatability and interobserver variability.

\section{IGG4-RELATED DISEASE}

IgG4-related disease (RD) is a systemic fibro-inflammatory disease characterized by elevated circulating levels of IgG4 and organ infiltration by $\mathrm{IgG}_{4}^{+}$plasma cells (PC). Any organ can be affected, pancreatic and biliary systems and salivary/lacrimal glands are the most frequently affected sites, the latter being involved in up to $50 \%$ of patients $(45,46)$. The spectrum of glandular involvement includes bilateral and symmetrical enlargement of both lacrimal and salivary glands, known as Mikulicz disease, unilateral submandibular gland involvement (formerly Küttner's tumour) and isolated involvement of lacrimal glands (45). The three histopathological hallmarks 
of IgG4-RD are dense lymphoplasmacytic infiltration, storiform fibrosis (an irregular cartwheel-like fibrotic pattern), and obliterative phlebitis. The inflammatory infiltrate includes both $\mathrm{T}$ and $\mathrm{B}$ lymphocytes with the latter organized in germinal centre-like structures and the former scattered within the lesion. In glandular tissues, the cellular infiltrate tends to surround ductal structures. A demonstration of $\mathrm{IgG}_{4}{ }^{+} \mathrm{PC}$ infiltrating the tissue is necessary for a pathological diagnosis. At least 10 or 50 (according to the organ) $\mathrm{IgG}^{+} \mathrm{PC}$ and a $\mathrm{IgG}^{+}{ }^{+} \mathrm{PC} / \mathrm{IgGG}^{+} \mathrm{PC}$ ratio $>40 \%$ ensure a sensitivity of $94.4 \%$ and a specificity of $86 \%(47,48)$. Ideally the biopsy should be taken from a major salivary gland, preferably from the submandibular glands (49). It should be noted that in IgG4-RD, symptoms of mucosal dryness are not as relevant as in pSS and objective tests to assess glandular secretion may yield normal results.

\section{AMYLOIDOSIS}

The term amyloidosis includes a heterogeneous group of diseases caused by the extracellular deposition of autologous fibrillar proteins (50). The key pathogenic event leading to amyloidosis is protein misfolding induced by several factors such as an increase of serum amyloid A protein (SAA) during chronic inflammation. The accumulation in the extracellular space of fibrils containing misfolded protein leads to progressive dysfunction of the target organ(s) (51). Virtually any organ can be targeted by amyloidosis. However, a certain tropism of some misfolded proteins to accumulate in specific organs has been described. The mechanisms underlying this tissue tropism are largely unknown (50). Amyloidosis can occur as limited or systemic disease and in the latter case about $60-80 \%$ of patients have detectable amyloid proteins in subcutaneous fat, rectal mucosa and MSG biopsy samples. The sensitivity of MSG biopsy $(83-100 \%)$ is higher compared to biopsies of the two other sites (57-85\%). However, subcutaneous fat biopsy ensures the highest specificity $(93 \%-100 \%)(5,52)$. H\&E staining of MSGs reveal amyloid de- posits as homogeneous and eosinophilic structures. Congo red staining allows identification of pathognomonic green birefringence of fibrils under cross-polarized light and represents the gold standard dye with which to differentiate amyloid from other protein deposits $(50,51)$. The distribution of lesions in MSGs may vary widely with the involvement of acini, ducts and vessels. It is interesting to note that Jamet et al. have recently demonstrated that in some subtypes of systemic amyloidosis, distinctive histological patterns of amyloid deposition in MSGs can be detected (53).

\section{SARCOIDOSIS}

Sarcoidosis is a chronic granulomatous disease that can affect any organs and tissues and has a heterogeneous clinical picture. The course of the disease as well as the prognosis is variable and depends on the affected site. More than half the patients are in remission within 3 years of diagnosis while up to one-third of patients have persistent disease, with progressive organ impairment $(54,55)$. Thoracic involvement occurs in more than $90 \%$ of patients with sarcoidosis with evidence of symmetrical hilar adenopathy at chest X-ray associated with general symptoms including fatigue, dyspnea and cough (56). Ear, nose and throat (ENT) manifestations are seen in approximately $10 \%$ to $15 \%$ of sarcoidosis patients and include head and neck lymphadenopathy, facial nerve palsy, and nasopharyngeal and laryngeal lesions. SG involvement is a rare manifestation $(57,58)$. Sarcoidosis of the SG presents as painless and persistent bilateral enlargement of the parotid glands with xerostomia and reduced salivary flow. Heerfordt's syndrome is defined as the concurrence of mild fever, bilateral parotid swelling, uveitis, and cranial nerve involvement, usually transient seventh nerve palsy $(59,60)$. Submandibular gland sarcoidosis has also been described and it can occur with or without concurrent parotid gland involvement (61). The histopathological feature of sarcoidosis is the presence of non-caseating granulomas that need to be differentiated from granulomas 
of other etiology (e.g. infectious diseases) (55). However, since granulomas are rather sparse within SGs, biopsies could be negative in up to $50 \%$ of patients (54).

\section{CONCLUSIONS}

The histological interpretation of SG biopsies provides a key clinical and research tool in several autoimmune diseases, including pSS, amyloidosis, sarcoidosis, and IgG4-related disease. Besides the diagnostic role, novel data highlight the prognostic value of histological features, making SG histopathology a potential biomarker for disease activity and response to treatment. Moreover, although evaluation with procedures alternative to biopsy, such as imaging modalities, should be encouraged, being less invasive, SG histology nevertheless provides biological proof of disease mechanisms and remains a milestone in clinical practice. Future studies aimed at standardizing sample scoring and interpretation of histopathological features as well as validation of the resulting protocols and recommendations, would be desirable.

\section{REFERENCES}

1. Miletich I. Introduction to salivary glands: structure, function and embryonic development. Front Oral Biol. 2010; 14: 1-20.

2. Brito-Zerón P, Baldini C, Bootsma $\mathrm{H}$, et al. Sjögren syndrome. Nat Rev Dis Primers. 2016; 2: 16047.

3. Vitali C, Bombardieri S, Jonsson R, et al. Classification criteria for Sjögren's syndrome: a revised version of the European criteria proposed by the American-European Consensus Group. Ann Rheum Dis. 2002; 61: 554-8.

4. Shiboski CH, Shiboski SC, Seror R, et al. 2016 American College of Rheumatology/European League Against Rheumatism classification criteria for primary Sjögren's syndrome: a consensus and data-driven methodology involving three international patient cohorts. Ann Rheum Dis. 2017; 76: 9-16.

5. Caporali R, Bonacci E, Epis O, et al. Safety and usefulness of minor salivary gland biopsy: retrospective analysis of 502 procedures performed at a single center. Arthritis Rheum. 2008; 59: 714-20

6. Pijpe J, Kalk WW, van der Wal JE, et al. Parotid gland biopsy compared with labial bi- opsy in the diagnosis of patients with primary Sjögren's syndrome. Rheumatology (Oxford). 2007; 46: 335-41.

7. Vitali C, Moutsopoulos HM, Bombardieri S. The European Community Study Group on diagnostic criteria for Sjögren's syndrome. Sensitivity and specificity of tests for ocular and oral involvement in Sjögren's syndrome. Ann Rheum Dis. 1994; 53: 637-4.

8. Guellec D, Cornec D, Jousse-Joulin S, et al. Diagnostic value of labial minor salivary gland biopsy for Sjögren's syndrome: a systematic review. Autoimmun Rev. 2013; 12: 416-20.

9. Greenspan JS, Daniels TE, Talal N, Sylvester RA. The histopathology of Sjögren's syndrome in labial salivary gland biopsies. Oral Surg Oral Med Oral Pathol. 1974; 37: 217-29.

10. Radfar L, Kleiner DE, Fox PC, Pillemer SR. Prevalence and clinical significance of lymphocytic foci in minor salivary glands of healthy volunteers. Arthritis Rheum. 2002; 47: 520-4.

11. Fisher BA, Brown RM, Bowman SJ, Barone F. A review of salivary gland histopathology in primary Sjögren's syndrome with a focus on its potential as a clinical trials biomarker. Ann Rheum Dis. 2015; 74: 1645-50.

12. Vitali C. Immunopathologic differences of Sjögren's syndrome versus sicca syndrome in $\mathrm{HCV}$ and HIV infection. Arthritis Res Ther. 2011; 13: 233.

13. Christodoulou MI, Kapsogeorgou EK, Moutsopoulos HM. Characteristics of the minor salivary gland infiltrates in Sjögren's syndrome. J Autoimmun. 2010; 34: 400-7.

14. Kapsogeorgou EK, Christodoulou MI, Panagiotakos DB, et al. Minor salivary gland inflammatory lesions in Sjögren syndrome: do they evolve? J Rheumatol. 2013; 40: 1566-71.

15. Zhou D, Chen YT, Chen F, et al. Critical involvement of macrophage infiltration in the development of Sjögren's syndrome-associated dry eye. Am J Pathol. 2012; 181: 753-60.

16. Goules AV, Kapsogeorgou EK, Tzioufas AG. Insight into pathogenesis of Sjögren's syndrome: Dissection on autoimmune infiltrates and epithelial cells. Clin Immunol. 2017 (Epub ahead of print).

17. Bombardieri M, Lewis M, Pitzalis C. Ectopic lymphoid neogenesis in rheumatic autoimmune diseases. Nat Rev Rheumatol. 2017; 13 : 141-54.

18. Chisholm DM, Mason DK. Labial salivary gland biopsy in Sjögren's disease. J Clin Pathol. 1968; 21: 656-60.

19. Tarpley TM, Anderson LG, White CL. Minor salivary gland involvement in Sjögren's syndrome. Oral Surg Oral Med Oral Pathol. 1974; 37: 64-74.

20. Daniels TE, Whitcher JP. Association of patterns of labial salivary gland inflammation 
with keratoconjunctivitis sicca. Analysis of 618 patients with suspected Sjögren's syndrome. Arthritis Rheum. 1994; 37: 869-77.

21. Liquidato BM, Duarte Barros M, Bussoloti Filho I, et al. Minor salivary glands morphology in xerostomia patients. Int $\mathrm{J}$ Morphol. 2006; 24: 489-93.

22. Daniels TE, Cox D, Shiboski CH, et al. Associations between salivary gland histopathologic diagnoses and phenotypic features of Sjögren's syndrome among 1,726 registry participants. Arthritis Rheum. 2011; 63: 2021-30.

23. Fisher BA, Jonsson R, Daniels T, et al. Standardisation of labial salivary gland histopathology in clinical trials in primary Sjögren's syndrome. Ann Rheum Dis. 2017; 76: 1161-8.

24. Vivino FB, Gala I, Hermann GA. Change in final diagnosis on second evaluation of labial minor salivary gland biopsies. J Rheumatol. 2002; 29: 938-44.

25. Risselada AP, Looije MF, Kruize AA, et al. The role of ectopic germinal centers in the immunopathology of primary Sjögren's syndrome: a systematic review. Semin Arthritis Rheum. 2013; 42: 368-76.

26. Carubbi F, Alunno A, Cipriani P, et al. Is minor salivary gland biopsy more than a diagnostic tool in primary Sjögren's syndrome? Association between clinical, histopathological, and molecular features: a retrospective study. Semin Arthritis Rheum. 2014; 44: 314-24.

27. Delli K, Haacke EA, Kroese FG, et al. Towards personalised treatment in primary Sjögren's syndrome: baseline parotid histopathology predicts responsiveness to rituximab treatment. Ann Rheum Dis. 2016; 75: 1933-8.

28. Risselada AP, Kruize AA, Goldschmeding R, et al. The prognostic value of routinely performed minor salivary gland assessments in primary Sjögren's syndrome. Ann Rheum Dis. 2014; 73: 1537-40.

29. Delli K, Haacke EA, Ihrler S, et al. Need for consensus guidelines to standardize the assessment of germinal centres and other histopathological parameters in salivary gland tissue of patients with primary Sjögren's syndrome. Ann Rheum Dis. 2016; 75: e32.

30. Bombardieri M, Barone F, Humby F, et al. Activation-induced cytidine deaminase expression in follicular dendritic cell networks and interfollicular large B cells supports functionality of ectopic lymphoid neogenesis in autoimmune sialoadenitis and MALT lymphoma in Sjögren's syndrome. J Immunol. 2007; 179: 4929-38.

31. Jonsson MV, Skarstein K. Follicular dendritic cells confirm lymphoid organization inthe minor salivary glands of primary Sjögren's syndrome. J Oral Pathol Med. 2008; 37: 515-21.

32. Cornec D, Jousse-Joulin S, Pers JO, et al. Contribution of salivary gland ultrasonography to the diagnosis of Sjögren's syndrome: toward new diagnostic criteria? Arthritis Rheum. 2013; 65: 216-25.

33. Reksten TR, Jonsson MV, Szyszko EA, et al. Cytokine and autoantibody profiling related to histopathological features in primary Sjögren's syndrome. Rheumatology (Oxford). 2009; 48: 1102-6.

34. Delaleu N, Mydel P, Kwee I, et al. High fidelity between saliva proteomics and the biologic state of salivary glands defines biomarker signatures for primary Sjögren's syndrome. Arthritis Rheumatol. 2015; 67: 1084-95.

35. Theander E, Vasaitis L, Baecklund E, et al. Lymphoid organisation in labial salivary gland biopsies is a possible predictor for the development of malignant lymphoma in primary Sjögren's syndrome. Ann Rheum Dis. 2011; 70: 1363-8.

36. Cornec D, Costa S, Devauchelle-Pensec V, et al. Blood and salivary-gland BAFF-driven Bcell hyperactivity is associated to rituximab inefficacy in primary Sjögren's syndrome. J Autoimmun. 2016; 67: 102-10.

37. Haldorsen K, Moen K, Jacobsen H, et al. Exocrine function in primary Sjögren syndrome: natural course and prognostic factors. Ann Rheum Dis. 2008; 67: 949-54.

38. Bookman AA, Shen H, Cook RJ, et al. Whole stimulated salivary flow: correlation with the pathology of inflammation and damage in minor salivary gland biopsy specimens from patients with primary Sjögren's syndrome but not patients with sicca. Arthritis Rheum. 2011; 63: 2014-20.

39. Carubbi F, Alunno A, Cipriani P, et al. A retrospective, multicenter study evaluating the prognostic value of minor salivary gland histology in a large cohort of patients with primary Sjögren's syndrome. Lupus 2015; 24 : 315-20.

40. Quartuccio L, Salvin S, Fabris M, et al. BLyS upregulation in Sjögren's syndrome associated with lymphoproliferative disorders, higher ESSDAI score and B-cell clonal expansion in the salivary glands. Rheumatology (Oxford). 2013; 52: 276-81.

41. Haacke EA, van der Vegt B, Vissink A, et al. Germinal centres in diagnostic labial gland biopsies of patients with primary Sjögren's syndrome are not predictive for parotid MALT lymphoma development. Ann Rheum Dis. 2017 (Epub ahead of print).

42. Alunno A, Carubbi F, Giacomelli R, Gerli R. The challenge to interpret conflicting results and the need of a univocal definition for germinal centers in primary Sjögren's syndrome. Ann Rheum Dis. 2017 (In press).

43. Carubbi F, Cipriani P, Marrelli A, et al. Efficacy and safety of rituximab treatment in early primary Sjögren's syndrome: a prospective, 
multi-center, follow-up study. Arthritis Res Ther. 2013; 15: R172.

44. Adler S, Korner M, Forger F, et al. Evaluation of histologic, serologic, and clinical changes in response to abatacept treatment of primary Sjögren's syndrome: a pilot study. Arthritis Care Res (Hoboken). 2013; 65: 1862-8.

45. Yamamoto M, Takahashi H, Shinomura Y. Mechanisms and assessment of IgG4-related disease: lessons for the rheumatologist. Nat Rev Rheumatol. 2014; 10: 148-59.

46. Stone JH, Zen Y, Deshpande V. IgG4-related disease. N Engl J Med. 2012; 366: 539-51.

47. Abraham M, Khosroshahi A. Diagnostic and treatment workup for IgG4-related disease. Expert Rev Clin Immunol. 2017: 1-9.

48. Masaki Y, Kurose N, Yamamoto M, et al. Cutoff values of serum IgG4 and histopathological IgG4+ plasma cells for diagnosis of patients with IgG4-related disease. Int J Rheumatol. 2012; 2012: 580814.

49. Witte T, Schulze-Koops H. IgG4-related sialadenitis: IgG4 is helpful, but biopsies are still crucial. Arthritis Res Ther. 2015; 17: 368.

50. Gillmore JD, Hawkins PN. Pathophysiology and treatment of systemic amyloidosis. Nat Rev Nephrol 2013; 9: 574-86.

51. Real de Asúa D, Costa R, Galván JM, et al. Systemic AA amyloidosis: epidemiology, diagnosis, and management. Clin Epidemiol. 2014; 6: 369-77.

52. van Gameren I, Hazenberg BP, Bijzet J, van Rijswijk MH. Diagnostic accuracy of subcutaneous abdominal fat tissue aspiration for detecting systemic amyloidosis and its utility in clinical practice. Arthritis Rheum. 2006; 54: 2015-21.

53. Jamet MP, Gnemmi V, Hachulla É, et al. Distinctive patterns of transthyretin amyloid in salivary tissue: a clinicopathologic study of 92 patients with amyloid-containing minor salivary gland biopsies. Am J Surg Pathol. 2015; 39: 1035-44.

54. Valeyre D, Prasse A, Nunes H, et al. Sarcoidosis. Lancet. 2014; 383: 1155-67.

55. Iannuzzi MC, Rybicki BA, Teirstein ASN. Sarcoidosis. N Engl J Med. 2007; 357: 2153-65.

56. Iannuzzi MC, Fontana JR. Sarcoidosis: clinical presentation, immunopathogenesis, and therapeutics. JAMA. 2011; 305: 391-9.

57. Surattanont F, Mandel L, Wolinsky B. Bilateral parotid swelling caused by sarcoidosis. J Am Dent Assoc. 2002; 133: 738-41.

58. Mandel L, Surattanont F. Bilateral parotid swelling: a review Oral Surg Oral Med Oral Pathol Oral Radiol Endod. 2002; 93: 221-37.

59. Vairaktaris E, Vassiliou S, Yapijakis C, et al. Salivary gland manifestations of sarcoidosis: report of three cases J Oral Maxillofac Surg. 2005; 63: 1016-21.

60. Ungprasert P, Crowson CS, Matteson EL. Clinical characteristics of parotid gland sarcoidosis: a population-based study. JAMA Otolaryngol Head Neck Surg. 2016; 142: 503-4.

61. Vourexakis Z, Dulguerov P, Bouayed S, et al. Sarcoidosis of the submandibular gland: a systematic review. Am J Otolaryngol. 2010; 31: 424-8. 